\title{
Sedimentation of Superfine Aerosol by Means of Ultrasound
}

\author{
Alexandra Antonnikova, Natalya Korovina, Olga Kudryashova \\ Institute for Problems of Chemical \& Energetic Technologies of the Siberian Branch of Russian Academy of Sciences, Biysk, Russia \\ Email: olgakudr@inbox.ru
}

Received July 16, 2013; revised August 18, 2013; accepted August 25, 2013

Copyright (C) 2013 Alexandra Antonnikova et al. This is an open access article distributed under the Creative Commons Attribution License, which permits unrestricted use, distribution, and reproduction in any medium, provided the original work is properly cited.

\begin{abstract}
The article is devoted to a problem of sedimentation of superfine aerosols with a characteristic size of particles, an order of values of micrometers. Acceleration of the sedimentation process is promoted by imposing of an acoustic field that leads to acceleration of coagulation of particles. Besides, pressure of the sound radiation directed down, accelerates gravitational sedimentation of an aerosol. The mathematical model is based on the Smolukhovsky's equation, describeing dynamics of change of distribution function by the sizes of aerosols particles taking into account ultrasonic influence, evaporation (for liquid aerosols) and sedimentation. Influence of introduction of an additional phase on coagulation and sedimentation speed is investigated. Results of a theoretical and experimental study of dispersion parameters and the sedimentation speed of superfine aerosols are presented.
\end{abstract}

Keywords: Aerosol Coagulation; Ultrasonic Influence; Two-Phase Aerosol; Evaporation of Droplets; Distribution Function of Particles by the Sizes

\section{Introduction}

Ability of sound waves to cause integration of the smallest particles weighed in air was found at an early stage of studying of features of strong ultrasound [1,2]. Acoustic waves, generally sound frequencies [3] are successfully used in sets of purification of gases and sedimentation of smokes. It is shown [4-6] that at sedimentation of the fine smokes (most difficult in this sense) it is necessary to use higher, ultrasonic frequencies. In this work, mechanisms of influence of ultrasonic waves on sedimentation of aerosols with a characteristic size of particles, a micrometer order are investigated in detail. The mathematical model for the description of kinetics of coagulation depending on key parameters of ultrasonic influence, properties of an aerosol and environment (frequencies and amplitudes of sound, concentration and disperse structure of an initial aerosol, viscosity and temperature of the environment, physical and chemical parameters of a material of particles) is offered. As option the problem of sedimentation of a two-phase aerosol with application and without application of ultrasonic influence is considered. Such task in practice takes place, for example, in mines (a coal dust and a water fog), at suppression of fires (a smoke and water), at sedimentation industrial dusts by means of water aerosols, etc.

\section{Mathematical Model}

Let's consider transformation of distribution by the sizes of particles of a cloud with the time $t$. Following $[5,6]$, write the balance equation (the Smolukhovsky's equation, integral case), describing change over time a vector of mass function of distribution of particles by the sizes:

$$
\frac{\partial g(D, t)}{\partial t}=I_{1}+I_{2}+I_{3} .
$$

where $I_{1}$ describes a decrease of particles with a diameter $D$ for a unit of time in a unit of volume because of collision of a drop of diameter $D$ with any drop of diameter $D_{1}$ :

$$
I_{1}=-g(D, t) \int_{0}^{D_{c r}(t)} K\left(D, D_{1}\right) g\left(D_{1}, t\right) \mathrm{d} D_{1},
$$

where $K\left(D, D_{1}\right)$ — probability of collisions of particles,

$$
D_{c r}=\sqrt{18 \eta H /\left(G \rho_{p} t\right)},
$$

$H$-the upper bound of a cloud; $G$-acceleration of gravity, $\eta$-dynamic viscosity of the environment, $\rho_{p}$ particle density. All particles, which weight exceeds critical value $D_{c r}(t)$, drop out of a cloud and do not take further part in coagulation; the distribution of particles on 
each moment of time $t$ will be cut off on the right end because of sedimentation of large particles, and, gradually this border will be displaced towards smaller particles.

$I_{2}$ describes emergence of particles of diameter $D$ because of collision of particles with diameters $D_{1}$ and $D-D_{1}$ :

$$
I_{2}=\frac{1}{2} \int_{0}^{D} K\left(D-D_{1}, D_{1}\right) g\left(D_{1}, t\right) g\left(D-D_{1}, t\right) \mathrm{d} D_{1} .
$$

Initial conditions for the Equation (1): at $t=t_{0} g\left(D, t_{0}\right)$ $=g_{0}(D)$-initial distribution of particles by the sizes. To the description of function of distribution of particles by the sizes usually apply gamma distribution. Numerical function

$$
f_{0}(D)=a D^{\alpha} \exp (-b D),
$$

where $b, \alpha$-parameters of distribution, $a$-normalizing coefficient, is connected with mass function by the following expression: $g(D)=m / m_{10} f(D)$, where $m_{10}$ - the arithmetic mean mass of particles: $m_{10}=\int_{0}^{\infty} m f(D) \mathrm{d} D$.

One of statistical characteristics of a distribution function of particles by the sizes is a volumetric surface diameter $D_{32}=(\alpha+3) / \mathrm{b}$.

\subsection{Evaporation of Droplets}

$I_{3}$ describes reduction of weight of particles because of their evaporation:

$$
\begin{aligned}
I_{3} & =\frac{\partial}{\partial m}\left(\frac{\mathrm{d} m}{\mathrm{~d} t} f(D)\right) \\
& =\frac{\partial}{\partial m}\left[\frac{2 \pi D_{f} M\left(p_{\text {drop }}-p_{p l}\right) f(D)}{R T}\right]
\end{aligned}
$$

where $m$ - the mass of a droplet; $D_{f}$-diffusion coefficient; $M$ - the molecular mass of a liquid droplet; $R$ - absolute gas constant; $T$-absolute temperature; $p_{\text {drop }}$ and $p_{p l}$ partial pressure over a droplet surface and a flat surface.

Considering Kelvin's formula

$$
\ln \left(p_{\text {drop }} / p_{p l}\right)=\frac{4 \sigma M}{\rho_{p} R T D},
$$

where $\sigma$ is a surface tension, expressing the mass of a particle through its diameter, we receive:

$$
I_{3}=\frac{\partial}{\partial D}\left[4 \pi D_{f} M p_{p l}\left(\exp \left(\frac{4 \sigma M}{\rho_{p} R T D}\right)-1\right) \frac{f(D)}{R T D \rho_{p}}\right] \text {. }
$$

\subsection{Probability of Collisions of Particles at Ultrasonic Influence}

A important question is definition of probability of colli- sions of particles: the higher is the collision probability, the quicker is coagulation and aerosol sedimentation. In lack of any influence, this size is caused by Brownian motion. In Model (1), probability of collisions of aerosol particles without additional influence was considered to the proportional sum of squares of diameters of particles:

$$
K\left(D, D_{1}\right)=\frac{k_{b} n_{0}}{v}\left(D^{2}+D_{1}^{2}\right)
$$

where $k_{b}$-proportionality coefficient; $v$ - kinematic viscosity coefficient of the environment. In the conditions of ultrasonic influence the probability of collisions of particles increases. Expression (2) has to include the parameters characterizing ultrasonic influence, first of all, amplitude and frequency of radiation.

It is received [5] that the number of meetings of particles in a sound field is proportional to diameter of particles, a square of speed of their movement $U_{0}$ (which, in turn, is defined by amplitude of sound waves), to concentration of particles $n_{0}$, streamline flow coefficient $k_{f l w}$ and in inverse proportion to viscosity of the environment $v$ :

$$
N \approx \frac{U_{0}^{2} n_{0} k_{f l w}^{2}\left(D^{2}+D_{1}^{2}\right)}{v} .
$$

The particle weighed in gas under the influence of forces of a sound field is involved in an oscillating motion. Depending on properties of the environment, the sizes and density of particles it can be involved by environment better or worse that is defined by involvement coefficient $k_{i n v}$ - the relation of amplitude of speed of the weighed particle to amplitude of speed of a particle of gas. Considering that between a particle and an environment Stokes strength works, we will receive a formula for involvement coefficient in the form of [7]:

$$
k_{i n v}=\frac{1}{\sqrt{1+\omega^{2} \tau^{2}}},
$$

where $\omega$ is the frequency of acoustic influence; $\tau=$ $\rho_{\mathrm{p}} D^{2} / 18 \eta$ is the time of a Stokes relaxation of a particle, $\eta$ is coefficient of dynamic viscosity of the environment.

Considering expression for involvement coefficient of a particle in a sound field (4), it is possible to consider that the particle is involved by a sound field, increasing probability of collisions with the fixed particle in $k_{f l w}^{2}=k_{a}\left(1-k_{i n v}\right)^{2}$ time, where $k_{a}$-proportionality coefficient. Then the probability of collisions of particles with diameters $D$ and $D_{1}$ is proportional to $N\left(1-k_{\text {inv }}\right)^{2}$ or taking into account (2)-(4):

$$
K\left(D, D_{1}\right)=\frac{k_{b} n_{0}}{v}\left(D^{2}+D_{1}^{2}\right)\left(1+k_{a} U_{0}^{2}\left(1-\frac{1}{\sqrt{1+\omega^{2} \tau^{2}}}\right)^{2}\right) .
$$


From (5) follows: the less is the diameter of particles (and $\tau$ ), the more frequency $\omega$ is necessary for increase of speed of coagulation. From this expression, it is also visible that increase of concentration of particles $n_{0}$ promotes coagulation and sedimentation acceleration. So it is possible to increase concentration of particles by introduction in an existing aerosol of an additional aerosol phase. In this case, it is necessary to expect that when using the same weight of an entered aerosol the bigger effect will be gained at a finer dispersion of the additional phase: it is more quantity of particles of $n_{0}$, the specific surface is higher, the number of collisions in unit of time, and higher the speed of coagulation and sedimentation.

The model of coagulation of an aerosol described above (1) for the account of the second phase, we will add with an initial condition as this:

$$
f(D)=a\left((1-\delta) D^{\alpha} \exp (-b D)+\delta D^{\alpha_{1}} \exp \left(-b_{1} D\right)\right),
$$

where distribution parameters with an index 1 belong to an additional phase of an aerosol; $\delta$ is a numerical part of particles of an additional phase. The probability of collisions increases at introduction of an additional phase on $1+\delta /(1-\delta)$ :

$$
K\left(D, D_{1}\right)=\frac{k_{b} n_{0}}{v}\left(1+\frac{\delta}{1-\delta}\right)\left(D^{2}+D_{1}^{2}\right) .
$$

The Model (1) describes, first of all, change of a distribution function of aerosol particles at impact on it ultrasonic field, evaporation of particles, gravitational sedimentation and introduction of an additional phase. In practical sense, the question of reduction of weight of an aerosol over time is important.

\subsection{Sedimentation of Aerosol}

Expression of change of weight because of sedimentation can be received from Stokes law of movement of particles in air:

$$
\frac{\mathrm{d} h}{\mathrm{~d} t}=\frac{G \rho_{p} D^{2}}{18 \eta},
$$

where $\mathrm{d} h$ is the distance which a particle of diameter $D$ passes during the time $\mathrm{d} t$. In each moment of time $d t$ from the mass of the aerosol placed in volume of $S H$, the mass of the particles placed in a layer $\mathrm{d} m=\mathrm{d} h(D) S$, where $S$ is the area of a bottom of the experimental camera will disappear. Relative reduction of weight will make

$$
\frac{\mathrm{d} m}{m_{\text {all }}}=\frac{\mathrm{d} h(D)}{H}
$$

where $m_{\text {all }}$ is the mass of an aerosol at the moment time. Thus,

$$
\frac{\mathrm{d} m}{\mathrm{~d} t}=\frac{\mathrm{d} h}{\mathrm{~d} t} \frac{m_{\text {all }}}{H}=\frac{G \rho_{p} D^{2} m_{\text {all }}}{18 \eta H} .
$$

It is also necessary to consider a weight decrease because of evaporation and because of those particles which completely dropped out in a sediment (particles with a diameter, more than $D_{c r}$ ). Thus, the general change of weight, after summation on all fractions of distribution, will be:

$$
\begin{aligned}
& \frac{\mathrm{d} m}{\mathrm{~d} t} \\
& =\int_{0}^{D_{c r}}\left(\frac{2 \pi D_{f} M D p_{p l}}{R T \rho_{p}}\left(e^{\frac{4 \sigma M}{\rho_{p} R T_{K} D}}-1\right)+\frac{G \rho_{p} D^{2} m_{\text {all }}}{18 \eta H}\right) g(D) \mathrm{d} D \\
& +\int_{D_{c r}}^{\infty} m_{\text {all }}(t) g(D) \mathrm{d} D .
\end{aligned}
$$

The second mechanism of increase of speed of sedimentation is that ultrasonic influence creates excessive pressure (at placement of a source of ultrasound over an aerosol cloud) [8]. This pressure leads to reduction of height of a cloud $H$ during radiation action, so, according to Expression (8), to acceleration of sedimentation of an aerosol (independently of its coagulation). Pressure of sound radiation is expressed by a formula [9]:

$$
P=2 E_{k 1}\left(1-R^{2}\right)-2 E_{k 2}
$$

where $E_{k 1}$ and $E_{k 2}$ are averages on time of value of density of kinetic energy of a falling wave in air and wave in a cloud, $R$ is reflection coefficient of a sound wave from an interface of phases. At level of intensity of $140 \mathrm{dBA}$, applied in installations for coagulation of aerosols, $P \sim 10$ $\mathrm{Pa}$. Speed of movement of the upper bound of the cloud caused by excessive radiation pressure, is defined by expression: $\frac{\mathrm{d} H}{\mathrm{~d} t}=\sqrt{2 P / \rho_{p}}$.

\section{Model Calculations and Comparison with the Experiment}

For creation of a liquid aerosol the atomizer (airbrush KRATON R 200 LVLP-02S) configured to necessary characteristics (the average size of particles about $9 \mathrm{mi}-$ crometers) was used. During carrying out experiments the camera was filled with an aerosol within 1 minute (the mass of the sprayed liquid made $9 \mathrm{~g}$ ). The additional phase (a water aerosol with parameters of distribution function $\alpha=2.1, b=0.9$ ) was generated by means of ultrasonic atomizer "Monsoon-2". For creation of the acoustic field influencing an aerosol, the ultrasonic disk radiator of ultrasonic devise "Solovey" was used. Technical characteristics of the ultrasonic device: diameter of 
a radiator $-320 \mathrm{~mm}$, level of sound pressure - not less than $140 \mathrm{dBA}$, the frequency of fluctuations $-28 \mathrm{kHz}$. Disperse characteristics and concentration of an aerosol measured by means of the optical methods described in [10] (devise LID-2M). The scheme of experimental installation is shown in Figure 1.

As the model environment sunflower oil is used. Sunflower oil contains water and flying substances about 10 to $30 \%$. In calculations, the part of fast-evaporated substances in oil was accepted, equal $15 \%$.

Dependence of relative weight of a sunflower oil aerosol $\mathrm{m} / \mathrm{m}_{0}$ is given in Figure 2(a) (where $m_{0}$ is the initial mass of particles of an aerosol), calculated by the model given above (curve) and measured experimentally (points), depending on time at ultrasonic influence (a curve 2) and without it (a curve 1). The inflection of a curve in the areas, about 100 seconds is caused by evaporation of water and flying substances from oil drops. Further evolution of an aerosol is caused by coagulation of drops, radiation pressure upon a cloud and sedimentation of particles. In Figure 2(b), dependence of volume and volumetric surface diameter on time for an oil aerosol is given in a case of ultrasonic and without ultrasonic influences. Distribution of particles from time is deformed poorly, but is slightly stronger when imposing an acoustic field that is connected with the accelerated coagulation of particles.

In Figure 3, dependence of relative weight (a) and the volumetric surface diameter (b) of an aerosol of sunflower oil from time is shown at ultrasonic influence with use of an additional phase (a water aerosol, a curve 2) and without it (a curve 1). Introduction in an aerosol of an additional phase accelerates aerosol sedimentation, promoting coagulation.

Figure 4 illustrates effect of influence of an additional phase of a water aerosol on sedimentation of sunflower oil without imposing of an acoustic field. Deeper de-

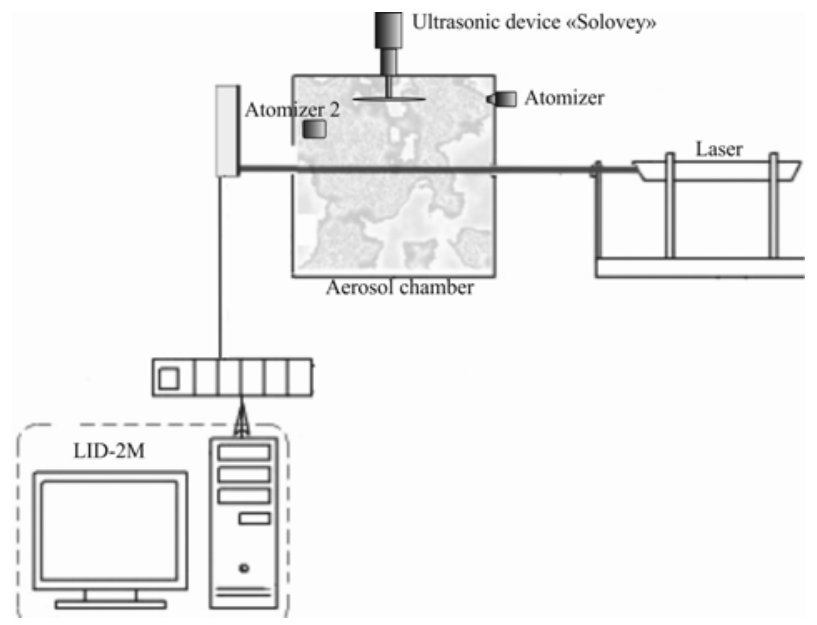

Figure 1. Scheme of experimental installation. crease in concentration of an aerosol is caused by evaporation of an additional phase of water droplets; then decrease in concentration happens, practically, with the same speed, as at an initial oil fog.

The estimated time of sedimentation of an oil aerosol is given in the Table $\mathbf{1}$ for four cases: without any influence, with ultrasonic influence, at ultrasonic influence and an additional water aerosol, only with a water aerosol, without ultrasonic.

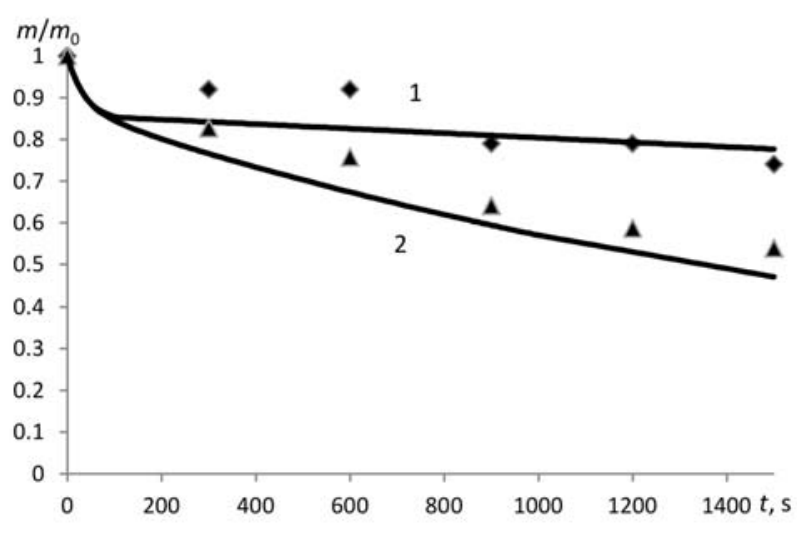

(a)

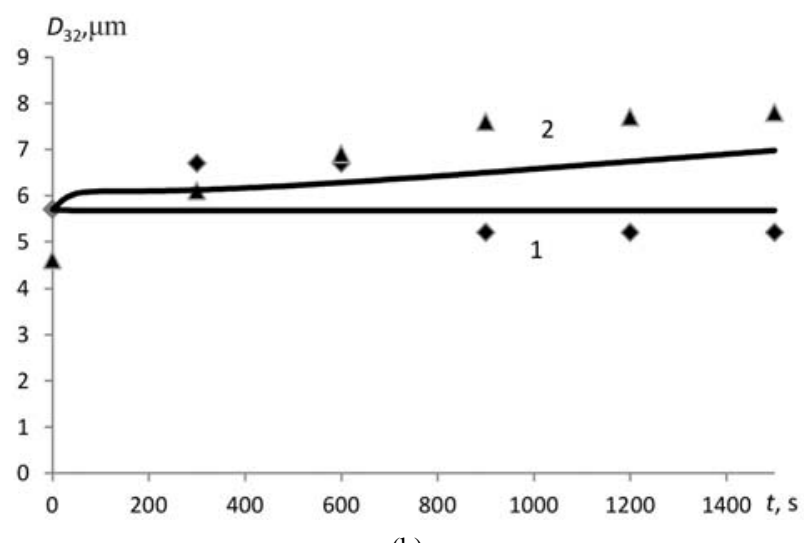

(b)

Figure 2. Dynamics of relative weight (a) and the volumetric surface diameter; (b) of an aerosol of sunflower oil without ultrasonic influence (1) and at ultrasonic influence (2).

Table 1. Time of full sedimentation of an oil aerosol at different types of influence.

\begin{tabular}{cc}
\hline Type of influence & Time of sedimentation, hours \\
\hline Without influence & 4.41 \\
Ultrasonic influence, & 1.07 \\
$28 \mathrm{kHz}, 140 \mathrm{dBA}$ & \\
$\begin{array}{c}\text { Addition water aerosol } \\
\left(D_{32} \sim 5 \text { micrometers }\right)\end{array}$ & 4.38 \\
Ultrasonic influence + addition \\
$\quad$ water aerosol
\end{tabular}




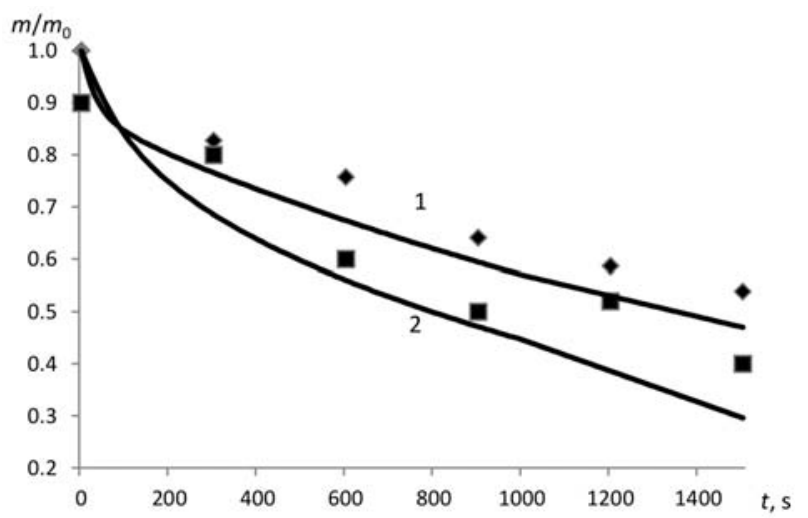

(a)

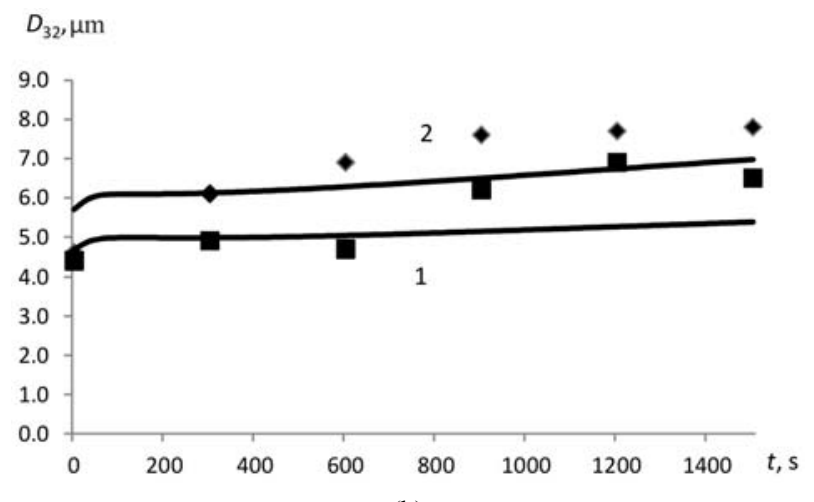

(b)

Figure 3. Dynamics of relative weight (a) and the volumetric surface diameter; (b) of an aerosol of sunflower oil at ultrasonic influence with an additional phase of a water aerosol (2) and without an additional phase (1).

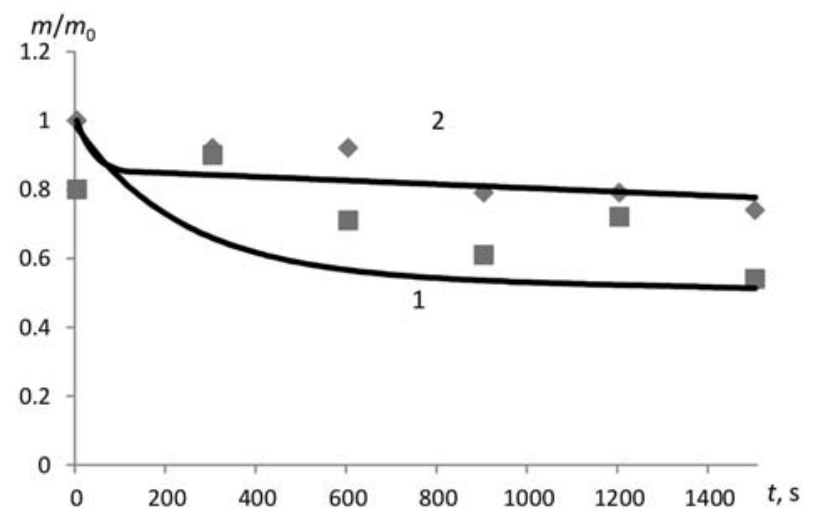

Figure 4. Dynamics of relative mass concentration of particles of an aerosol of sunflower oil without ultrasonic influence: 1 -with introduction of $5 \mathrm{~g}$ of a water aerosol in addition; 2-without introduction of an additional aerosol.

\section{Conclusion}

Apparently from the carried-out calculations, ultrasonic influence and introduction of an additional superfine wa- ter aerosol allows to lower time of sedimentation of an initial (non-evaporated) aerosol significantly. Experiments confirm this conclusion. The offered physicomathematical model based on the equation of Smolukhovsky taking into account evaporation, acceleration of coagulation and cloud sedimentation at the expense of ultrasonic influence, including, clouds of a two-phase aerosol, describes experimental data well.

\section{REFERENCES}

[1] H. S. Patterson, W. Cawood, "Phenomena in a Sounding Tube," Nature, Vol. 127, No. 3209, 1931, p. 667.

[2] H. St. Clair, "Agglomeration of Smoke, Fog or Dust Particles by Sonic Waves," Industrial \& Engineering Chemistry Research, Vol. 41, No. 11, 1949, p. 2434. doi:10.1021/ie50479a022

[3] B. Maczewski-Rowinski, "Precipitation of Dust From Industial WasteGases by Means of the C.I.W.P. Siren," Proceedings of the II Conference on Ultrasonics, PWN, Warsawa, 1957, pp. 23-29.

[4] V. N. Khmelev, A. V. Shalunov and K. V. Shalunova, "The Acoustical Coagulation of Aerosols," Proceedings International Workshop and Tutorial on Electron Devices and Materials, Novosibirsk, 1-5 July 2008, pp. 289-294.

[5] A. A. Antonnikova, O. B. Kudryashova, K. V. Shalunova, "Application of Ultrasound to Settle submicron Aerosols," Proceeding International Conference and Seminar on Micro/Nanotechnologies and Electronic Devices EDM, Biysk, 2012, pp. 87-89.

[6] A. A. Antonnikova, N. V. Korovina, O. B. Kudryashova and I. R. Akhmadeev, "Experimental Study of the Processes of Aerosol Transformation under Ultrasonic Impact," Atmospheric and Oceanic Optics, Vol. 26, No. 1, 2013, pp. 57-59. doi:10.1134/S102485601301003X

[7] C. W. Oseen, "The Theory of Liquid Crystals," Transactions of the Faraday Society, Vol. 29, No. 140, 1933, pp. 883-889. doi:10.1039/tf9332900883

[8] P. I. Westervelt, "Acoustic Radiation Pressure," JASA, Vol. 29, No. 1, 1957, p. 26.

[9] J. W. Strutt Baron Rayleigh, "The Theory of Sound," Macmillan and Company, New, York, 1894.

[10] O. Kudryashova, A. Pavlenko, B. Vorozhtsov, S.Titov, V. Arkhipov, S. Bondarchuk, E. Maksimenko, I. Akhmadeev and E. Muravlev, "Remote Optical Diagnostics of Nonstationary Aerosol Media in a Wide Range of Particle sizes," In: Photodetectors, InTech, Rijeka, 2012, pp. 341364.

http://www.intechopen.com/books/photodetectors/remote -optical-diagnostics-of-nonstasionary-aerosol-media-in-awide-range-of-particle-sizes 